\title{
A THEOREM OF RADÓ'S TYPE FOR THE SOLUTIONS OF A QUASI-LINEAR EQUATION
}

\author{
Petri Juutinen And Peter Lindqvist
}

\begin{abstract}
In this note, we prove a Radó type theorem for the solutions of the so-called $p$-harmonic equation

$$
\operatorname{div}\left(|\nabla u|^{p-2} \nabla u\right)=0
$$

in any dimension. We show that a function $u \in C^{1}(\Omega)$ which is $p$-harmonic in $\Omega \backslash\{x: u(x)=0\}$ is indeed $p$-harmonic in the whole domain $\Omega$. Our proof relies on a viscosity solution characterization of $p$-harmonic functions obtained in [JLM].
\end{abstract}

The classical theorem of T. Radó says that, if the continuous complex function $f(z)$ is analytic when $f(z) \neq 0$, then it is analytic in its whole domain of definition. This result has been generalized in many ways. We are interested in the corresponding phenomenon for solutions of partial differential equations. J. Král [Kr1] obtained the following theorem for harmonic functions defined in a domain $\Omega$ in $\mathbb{R}^{n}$.

Theorem (Král). Let $u \in C^{1}(\Omega)$. If the function $u$ is harmonic in the set

$$
\Omega \backslash\{x \in \Omega: u(x)=0\},
$$

then $u$ is harmonic in the whole $\Omega$.

The same theorem holds with the Laplace equation replaced by any linear elliptic equation of the second order with constant coefficients, cf. [Kr2]. The objective of this note is to extend Král's theorem to the so-called $p$-harmonic equation

$$
\operatorname{div}\left(|\nabla u|^{p-2} \nabla u\right)=0,
$$

where $1<p<\infty$. This is the Euler-Lagrange equation for the variational integral

$$
\int_{\Omega}|\nabla u|^{p} d x
$$

2000 Mathematics Subject Classification. Primary: 35J70, 35B60; Secondary: 32D20.

Key words and phrases. p-Laplacian, Radó's theorem, viscosity solutions.

Received June 19, 2002. 
Notice that for $p=2$ we have the Dirichlet integral and the Laplace equation. The solutions of equation (1) are called $p$-harmonic functions and they will be defined below. We will prove the following theorem.

3. Theorem. Let $\Omega$ be a domain in $\mathbb{R}^{n}$ and suppose that $u \in C^{1}(\Omega)$. If $u$ is a solution to the equation (1) in the open set $\Omega \backslash\{x: u(x)=0\}$, then it is a solution in the whole $\Omega$.

This theorem was proved by T. Kilpeläinen [Ki] in the plane, where he could use the fact that the complex gradient

$$
\frac{\partial u}{\partial x}-i \frac{\partial u}{\partial y}
$$

of a $p$-harmonic function $u$ is a quasiregular mapping. No such information is available in space. The whole difficulty comes from the critical points: the equation degenerates badly when $\nabla u=0$. The crucial point is to establish the following result.

4. Proposition. Let $u \in C^{1}(\Omega)$. If $u$ is p-harmonic in

$$
\Omega \backslash\{x \in \Omega: \nabla u(x)=0\},
$$

then $u$ is p-harmonic in the whole $\Omega$.

Our way to deal with the critical points comes from a rather unexpected direction: the theory of viscosity solutions. In [JLM], the $p$-harmonic functions, originally defined as the continuous weak solutions in the distributive sense, were characterized as viscosity solutions in such a way that the critical points can, to a certain extent, be neglected. This is exactly what is needed to prove the above proposition. In the plane case we provide an alternative to Kilpeläinen's proof. It is worth mentioning that our proof is, as it were, new even for the Laplace equation.

Let us finally mention that the assumption about continuous differentiability for the function cannot be weakened. Simple, essentially one-dimensional, examples show that mere Lipschitz continuity will not do. It is likely that our method also applies to more general quasi-linear equations

$$
\operatorname{div} A_{p}(x, \nabla u)=0
$$

where $A_{p}(x, \xi) \cdot \xi \approx|\xi|^{p}$. However, here the dependence on $x$ must be rather restricted so that $\operatorname{div} A_{p}(x, \nabla \varphi)$ can be evaluated pointwise for a smooth $\varphi$, at least when $\nabla \varphi(x) \neq 0$. We will return to this issue in a subsequent paper.

To be on the safe side we recall the definition of $p$-harmonic functions.

5. Definition Let $u \in C(\Omega) \cap W_{l o c}^{1, p}(\Omega)$. We say that $u$ is $p$-harmonic in the open set $\Omega$, if

$$
\int_{\Omega}|\nabla u|^{p-2} \nabla u \cdot \nabla \eta d x=0
$$


whenever $\eta \in C_{0}^{\infty}(\Omega)$.

It is known that the $p$-harmonic functions are of class $C_{l o c}^{1, \alpha}(\Omega)$, where $\alpha=$ $\alpha(n, p)$. In order to prove the proposition, we need an equivalent definition. In $[\mathrm{JLM}]$ we defined the viscosity $p$-supersolutions of the equation (1). For a continuous function the definition takes the following simple form.

6. Definition We say that $u \in C(\Omega)$ is a viscosity $p$-supersolution if, whenever $x_{0} \in \Omega$ and $\varphi \in C^{2}(\Omega)$ are such that

(1) $u\left(x_{0}\right)=\varphi\left(x_{0}\right)$,

(2) $u(x)>\varphi(x)$, when $x \neq x_{0}$,

(3) $\nabla \varphi\left(x_{0}\right) \neq 0$,

we have

$$
\operatorname{div}\left(\left|\nabla \varphi\left(x_{0}\right)\right|^{p-2} \nabla \varphi\left(x_{0}\right)\right) \leq 0
$$

Notice that the test-function $\varphi$ touches $u$ from below at the point $x_{0}$. Notice also the important fact that, if it so happens that $\nabla \varphi\left(x_{0}\right)=0$, then there is no requirement at all. In this respect our definition is different from the conventional one. The conventional definition (see e.g. [CIL]), valid for $p \geq 2$, makes no exemption at the critical points. We take full advantage of the less demanding requirements above. Now the viscosity $p$-subsolutions are defined in an analogous way, see [JLM].

7. Theorem. Let $u \in C(\Omega)$. Then $u$ is p-harmonic if and only if $u$ is both $a$ viscosity p-supersolution and a viscosity p-subsolution.

Proof. This is Corollary 2.6 in [JLM].

Proof of the Proposition 4. In order to prove that the given function $u \in C^{1}(\Omega)$ is $p$-harmonic, we first prove that Definition 6 is satisfied. Let $x_{0} \in \Omega$ and suppose that $\varphi$ is a test-function, touching $u$ from below at the point $x_{0}$. By the differential calculus,

$$
\nabla \varphi\left(x_{0}\right)=\nabla u\left(x_{0}\right)
$$

Hence, if $\nabla u\left(x_{0}\right)=0$, there is nothing to prove. On the other hand, if $\nabla u\left(x_{0}\right) \neq$ 0 , then it is already clear, according to Theorem 7 and the assumption, that $\operatorname{div}\left(\left|\nabla \varphi\left(x_{0}\right)\right|^{p-2} \nabla \varphi\left(x_{0}\right)\right) \leq 0$. Thus $u$ is a viscosity $p$-supersolution. Similarly, $u$ is seen to be a viscosity $p$-subsolution. Now Theorem 7 yields the desired result.

Since the solution concept is a local one, Theorem 3 follows from Proposition 4 and the lemma below. 
8. Lemma. Suppose that $u \in C^{1}(\Omega)$ is such that $\nabla u \neq 0$ in $\Omega$. If $u$ is $p$ harmonic in the set $\Omega \backslash\{x: u(x)=0\}$, then $u$ is p-harmonic in the whole $\Omega$.

Proof. This is Proposition 8 in [Ki]. For the convenience of the reader, we comment on the proof. Since the problem is local one we may assume that $|\nabla u| \geq \varepsilon>0$. Then the set

$$
S=\{x \in \Omega: u(x)=0\}
$$

is a regular hypersurface. Locally it can be mapped onto the hyperplane

$$
H=\left\{\left(x_{1}, \ldots, x_{n}\right) \in \mathbb{R}^{n}: x_{n}=0\right\}
$$

with the aid of a bi-Lipschitz diffeomorphism. This transforms the problem to the corresponding problem for a certain equation

$$
\operatorname{div} A_{p}(x, \nabla v(x))=0
$$

but now $S$ has been replaced by the hyperplane $H$. That is, if $v(x)=0$, then $x \in H$. The proof is comparatively simple for a hyperplane and is given in Lemma 2.22 in $[\mathrm{M}]$. The essential phenomenon in that proof is a cancellation effect.

This concludes the proof of Theorem 3 .

\section{References}

[CIL] Crandall, M.G., H. Ishii, and P-.L. Lions, User's guide to viscosity solutions of second order partial differential equations, Bull. Amer. Math. Soc. 27 (1992), 1-67.

[JLM] Juutinen, P., P. Lindqvist, J. Manfredi, On the equivalence of viscosity solutions and weak solutions for a quasilinear equation, SIAM J. Math. Anal. 33 (2001), 699-717.

[Ki] Kilpeläinen, T., A Radó type theorem for p-harmonic functions in the plane, Electronic J. Differential Equations 9 (1994), 1-4.

[Kr1] Král, J., Some extension results concerning harmonic functions, J. London Math. Soc. (2) 28 (1983), 62-70.

[Kr2] Král, J., Extension results of the Radó type, Rev. Roumaine Math. Pures Appl. 36 (1991), 71-76.

[M] Martio, O., Counterexamples for unique continuation, Manuscripta Math. 60 (1988), $21-47$.

Department of Mathematics and Statistics, P.O.Box 35 (MaD), Fin-40014 UniverSITY OF JYVÄSkYLÄ, FinlAND

E-mail address: peanju@maths.jyu.fi

Department of Mathematics, Norwegian University of Science and Technology, N-7491 Trondheim, Norway

E-mail address: lqvist@math.ntnu.no 